\title{
Associations between stress and depression symptom profiles vary according to serotonin transporter polymorphism in rural Australians
}

This article was published in the following Dove Press journal:

Neuropsychiatric Disease and Treatment

Christopher F Sharpley'

Vicki Bitsika ${ }^{2}$

Mary E McMillan'

Emmanuel Jesulola'

Linda L Agnew'

'Brain-Behaviour Research Group, University of New England, Armidale, NSW, Australia; ${ }^{2}$ Centre for Autism Spectrum Disorders, Bond University, Gold Coast, QLD, Australia
Correspondence: Christopher F Sharpley Brain-Behaviour Research Group, University of New England, Armidale, NSW 235I, Australia

Tel +6I 267732596

Email csharpl3@une.edu.au
Purpose: Depression remains difficult to treat in all cases, and further investigation of the role of genetic and environmental factors may be valuable. This study was designed to investigate the association between the short (s) versus non-short (non-s) 5HTTLPR variants, presence of childhood stressors and recent life stressors, and depression, and to do so at two levels that would expose the associations between total depression scores and also individual depression items. Materials and methods: Two hundred and forty-nine volunteers from one of the Australian Electoral Office electorates covering a large rural land area completed a series of questionnaires about childhood and recent life stress and depression, and provided a buccal cell sample for genotyping the 5-HTTLPR polymorphism into $s$ versus non- $s$ carriers.

Results: Although there were no significant differences in the depression scores of the s-carriers versus the non-s carriers, each subtype of the 5-HTTLPR polymorphism showed different patterns of association between childhood stress and depression symptoms, and between recent life stress and depression symptoms.

Conclusion: Individualization of therapy for depression may be achieved through consideration of the specific associations that patients exhibit between life stress, 5-HTTLPR polymorphism, and depression symptomatology.

Keywords: genes, mental health, life experiences

\section{Introduction}

Despite being the major contributor to the total disease burden, ${ }^{1}$ having one of the highest costs of care among all chronic diseases, producing adverse effects on physical health, relationships, and cognitive performance, ${ }^{2}$ and resulting in suicide in about $15 \%$ of all patients, ${ }^{3}$ depression remains difficult to treat effectively in all cases. For example, several years ago, it was reported from the STAR*D study of over 4,000 depressed patients that initial treatments were successful in only $37 \%$ of patients, with second-line treatments achieving an additional $31 \%$ remission. ${ }^{4}$ As suggested by these data, there was often significant interpatient variability in outcomes. ${ }^{5}$ Possible reasons for this wide variety in treatment efficacy for depression include patients' different biologic vulnerabilities to depression and differences in their particular symptom profiles. ${ }^{6}$

\section{Genetic factors}

Biologic vulnerability to depression is reflected in the strong heritability for depression, estimated at $35 \%$. $^{7}$ Consequently, many studies have been undertaken to search for the specific genetic factors that may be related to an inherited vulnerability to depression. A great deal of that research has been focused upon the serotonin transporter gene 
SLC6A4 and one of its polymorphisms from the promoter region, known as 5-HTTLPR. SCL6A4 encodes the serotonin transporter protein 5-HTT, which carries serotonin (5-HT) away from the synapse after signal transmission for later reuse. In humans, SCL6A4 resides on chromosome 17q11.1 but its ability to encode 5-HTT is influenced by the presence of the promoter region 5-HTTLPR about $1 \mathrm{~kb}$ upstream of the 5-HTT gene transcription initiation region. 5-HTTLPR polymorphisms are grouped into "short" (s) or "long" (1) forms, referring to the number of base pairs in each form; the short form has 44 fewer base pairs than the long form. ${ }^{8,9}$ Humans may carry three possible genotypes, a combination of short and long ( $\mathrm{sl}$ ), or short and short (ss), or long and long (11) forms, although most research is focused upon the dichotomy of s-present versus s-absent (ie, ss, sl versus ll). The short (s) form polymorphism of 5-HTTLPR restricts the transcriptional activity of the promoter, producing low functional expression of SCL6A4 and hence reducing serotonin reuptake; the long (1) form does not have this effect.

Following Caspi et al's ${ }^{10}$ first report of the significant interaction between the 5HTTLPR, childhood stress, recent stressful life events, and depression, many studies and reviews of this association have been published, often with differing results. For example, Lopez-Leon et $\mathrm{al}^{11}$ cited 24 studies, 20 of which had an odds ratio $>1.0$ for the $s$ allele versus the 1 allele, but only three of those studies produced statistically significant differences between the two forms. Arguing in 2010 that the 5-HTTLPR does not have a simply unilateral relationship with depression but operates through the " $\mathrm{g} \times \mathrm{e}$ " interaction of genetic and environmental (stress) factors over the lifetime (including both Childhood Stressors and Recent Life Stressors), Risch et al ${ }^{12}$ and Munafo et $\mathrm{al}^{13}$ performed meta-analyses of the interaction between 5-HTTLPR, stress, and depression on 5 and 14 studies, respectively, and concluded that there was no evidence for the hypothesis. However, in a further review, Uher et $\mathrm{al}^{14}$ reviewed 34 studies of the interaction between 5-HTTLPR, environmental adversity, and depression, confirming that relationship. Some other meta-analyses ${ }^{15-17}$ raised doubts about the consistent association between the ss variant, stress, and depression. The current state of uncertainty regarding the association between the 5HTTLPR, stress, and depression is reflected in the conclusions of two most recent reviews, one of which found "a small but significant effect of 5-HTTLPR in interaction with stress in the prediction of depression."18 (p. 339) and the other of which reported that the association was "not broadly generalizable but must be of modest effect size and only observable in limited situations."19 (p. 133).
However, by their nature, meta-analyses combine data from many studies, potentially occluding the findings from some studies that show effects in a different direction to the majority. Those differences are not always attributable to methodologic defects. For example, although Karg et al ${ }^{15}$ found evidence that the ss allele of the 5-HTTLPR was associated with an increased risk of individuals who developed major depressive disorder (MDD) following childhood stressors, major medical conditions, and (less robust but still statistically significant) general life stressors, 15 of the 54 studies they reviewed reported no association between the ss, stress, and depression, and a further 6 reported at least some aspect of their sample in which carriers of the long (11) allele were more likely than ss carriers to become depressed following significant life stress ${ }^{15}$ (Table 1). Similarly, Sharpley et a $1^{17}$ also found that, of the 81 studies they reviewed, the significant relationship between the short form of the 5-HTTLPR, stress, and depression was confirmed ( $p=0.0000009$ ), but nearly $26 \%$ studies failed to show any significant association between the 5-HTTLPR, stress, and depression, and four studies found opposite results to those expected (ie, the association was between the 1 variant, stress, and depression rather than the $\mathrm{s}$ variant, stress, and depression). Examination of the methodologies of all those studies failed to identify any flaws in the opposite or unequivocal studies, and the latter had larger sample sizes than those studies that supported the expected association, arguing that the null results were not an outcome of insufficient statistical power. Thus, while meta-analytic data are of great value in determining overall findings or such associations as the 5HTTLPR, stress, and depression, there remains a valid role for individual studies of the particular characteristics of selected samples.

\section{Symptom profiles}

The current diagnosis of MDD covers nine possible symptoms, of which five must be present most of the time; of those five, one must be either sadness or anhedonia. ${ }^{3}$ The nine symptoms cover a wide range of observable behavior, from changes in dietary intake or sleeping patterns to difficulties

Table I Mean, SD, and range of scores for psychological variables, and distribution of 5 HTTLPR variants

\begin{tabular}{llll}
\hline Variable & Mean & SD & Range \\
\hline SDS total score & 37.91 & 8.50 & $21-74$ \\
Childhood Stressors & 67.76 & 9.28 & $28-56$ \\
Recent Life Stressors & 26.76 & 1.36 & $20-28$ \\
5HTTLPR variants & s-present=184 & s-absent=65 & \\
& $(73.9 \%)$ & $(26.1 \%)$ & \\
\hline
\end{tabular}

Abbreviation: SDS, Self-rating Depression Scale. 
concentrating or making decisions, and feeling worthless or having thoughts of death. There are also a large number of associated features for MDD, which are linked to the nine major criteria but describe slightly different depressive behaviors. ${ }^{3}$ This heterogeneity of symptomatology for MDD implies that there are many ways in which a patient might qualify for the MDD diagnosis, and an arithmetical analysis of the possible forms of diagnosis that meet the criteria for MDD revealed that there were 1,497 such possible ways of fulfilling the diagnosis of MDD. ${ }^{20}$ Further, analysis of symptom data from 3,703 depressed outpatients at the beginning of the first treatment stage of the STAR *D trial ${ }^{21}$ identified 1,030 unique symptom profiles, $83.9 \%$ of which were endorsed by five or fewer subjects and $48.6 \%$ of which were endorsed by only one individual. Severity of the total depression symptomatology did not reduce this high level of heterogeneity across the MDD symptoms, leading those authors to comment that "the analysis of individual symptoms, their patterns, and their causal associations will provide insights that could not be discovered in studies relying on only sum-scores" (p. 96).

A focus on the symptom profiles of depression entails looking beyond the dichotomous diagnosis of depression used in the Diagnostic and Statistical Manual of Mental Disorders, Fifth Edition, ${ }^{3}$ or the total score from a checklist or self-report inventory, to considering the entire range of depressive symptoms present in a particular patient. Previous studies have reported on the prevalence of various depression symptom profiles in general community samples ${ }^{22}$ as well as the association between those depression profiles and treatment outcomes. ${ }^{6}$ This perspective follows the call for "personalized medicine" approaches to the diagnosis and treatment of depression ${ }^{23}$ as a way of better matching diagnostic profiles to specific treatment options for more effective outcomes. However, to date, there have been no studies of the depressive profile of multiple MDD symptoms when investigating the association between the 5HTTLPR, stress, and depression; all previous studies relied on total scores from depression inventories or clinical evaluation of the presence/absence of MDD. These dichotomous measures are valid but do not provide insight into the more detailed symptom profiles of the participants in such studies and the possible relationships those symptom profiles may have with genetic factors such as the 5HTTLPR. Dichotomization of what are effectively linear scores across a range of symptoms and severities brings some limits to the power of the statistical analyses possible with such data and the results from those analyses. ${ }^{24}$ This limitation might have contributed to the disagreement in the previous literature regarding the existence of the 5HTTLPR-stress-depression association.

\section{Study aims}

Therefore, this study aimed to investigate the association between the ss versus non-s 5HTTLPR variants, presence of Childhood Stressors and Recent Life Stressors, and depression, and to do so at two levels that would expose the associations between total depression scores and also individual depression items. This process was designed to enable the information to be gathered regarding the depression profiles of the two subgroups of 5HTTLPR variant carriers and then compared for similarities and/or differences. The four research questions investigated were as follows: 1) Is there a significant difference in the severity of total depression across participants carrying the s-present versus s-absent variants of the 5HTTLPR? 2) Is there a significant difference in the severity of individual depression items across participants carrying the s-present versus s-absent variants of the 5HTTLPR? 3 ) Is there a difference in the total depression scores arising from the $\mathrm{g} \times \mathrm{e}$ association between the 5HTTLPR and total Recent Life Stressors or Childhood Stressors? and 4) Is there a difference in the depression symptom profiles arising from the $\mathrm{g} \times$ e association between the 5HTTLPR and total Recent Life Stressors or Childhood Stressors?

The specific population sampled was rural Australians, principally because many previous studies of these factors used urban samples; data from a selected rural sample can provide an additional insight into the 5HTTLPR-stressdepression association that has not previously been reported as such. In addition, depression has been reported at a prevalence rate of $10 \%$ in rural Australia, ${ }^{25,26}$ which is similar to that in urban Australia, but the suicide rate is several times higher in rural Australians than in urban Australians and has increased at a greater rate during recent years. ${ }^{27}$ There are also some data suggesting that gender is a confounder in the effect of the 5-HTTLPR upon depression, ${ }^{28}$ and even that males' depression may be associated with the 11 form, whereas females' depression is associated with the ss form. ${ }^{29}$ This confound with gender may account for some of the findings reported in the meta-analyses reviewed above and highlights the need to consider gender in studies of the 5-HTTLPR and depression.

\section{Materials and methods Participants}

Volunteers from one of the Australian Electoral Office electorates covering a large rural land area were invited by letter 
to participate in a study "about your mental health." The letter emphasized that individuals who had not experienced mental health problems were invited as much as those who had such experiences so that comparative data could be collected.

\section{Instruments}

\section{Background questionnaire}

Participants were asked to state their age (years) and sex.

\section{The Self-rating Depression Scale (SDS)}

The 20-item Zung $\operatorname{SDS}^{30}$ is based on the data from factor analytic studies of $\mathrm{MDD}^{31}$ and fits the most recent definitions of that disorder. ${ }^{3}$ Respondents indicate the frequency of each of those 20 items by answering "None or a little of the time", "Some of the time", "Good part of the time", or "Most or all of the time", which produce numerical scores of $1-4$, providing total raw scores from 20 to 80 . SDS raw scores of $\geq 40$ indicate the presence of "clinically significant depression". ${ }^{32}$ (p. 335). The SDS has demonstrated split-half reliability of $0.81,{ }^{30} 0.79,{ }^{33}$ and $0.94 .{ }^{34}$ Internal consistency (alpha) has been reported as 0.88 for depressed patients, 0.93 for nondepressed patients, ${ }^{35}$ and 0.84 for a previous Australian sample. ${ }^{36}$ The SDS has been shown to be superior to the MMPI Depression Scale and the Beck Depression Inventory for assessing depression in male psychiatric inpatients. ${ }^{35}$ SDS raw scores were used in this study.

Childhood Stressors were measured through a dichotomous (present/absent) response format to 29 questions including the content and structure of those used in previous major studies ${ }^{37}$ and the National Comorbidity Survey. ${ }^{38}$ Questions asked participants to state whether "any of the following happened to you when you were a child," and included content regarding (but not limited to) parental marital separation and divorce; experiences of neglect; physical, sexual, and mental abuse; parental unemployment; parental drug/alcohol abuse; suffering from a serious illness; mistreatment at school; family members' suicide or attempted suicide; and death of a parent. This content argues for the validity of the scale, and the internal consistency (Cronbach alpha) of this scale in the current study was 0.96 .

Recent Life Stressors were measured through dichotomous responses to the 14-item Recent Life Stress scale developed for the Hawaii Personality and Health Cohort Study, a population-based cohort participating in a longitudinal study of personality and health spanning 40 years from childhood to midlife. ${ }^{39}$ Participants were asked whether a series of stressful events had occurred to them during the past 6 months (for recency). These events included death or serious injury of a parent, partner, child, close relative, relationship breakdown, serious problems with relatives, close friends or neighbors, being unemployed or losing a job, having major financial problems, difficulties with the police or law, and losing something valuable, supporting its content validity. Internal consistency for this scale in the current study was 0.89 .

\section{DNA extraction}

Genomic DNA was extracted from mouthwash samples using a Gentra Puregene Buccal cell kit (Qiagen Inc., MD, USA). Mouthwash samples were centrifuged for 5 minutes at $2,000 \times g$ to pellet cells, and DNA was extracted following the manufacturer's protocol. Presence of intact, high-molecularweight DNA was confirmed through gel electrophoresis and quantified using a NanoDrop 8000 spectrophotometer (NanoDrop, ThermoFisher, Melbourne, Australia).

\section{HTTLPR genotyping}

5HTTLPR genotyping for short and long alleles into s-present (ie, ss, sl) versus s-absent (ie, 11) was carried out using polymerase chain reaction (PCR), as described by Wendland et al. ${ }^{40}$ The 5HTTLPR alleles were amplified using the following primers: forward $5^{\prime}$ TCCTCCGCTTTGGCGCC TCTTCG 3', reverse 5' TGGGGGTTGCAGGGGA GATCCTG $3^{\prime}$. These primers yield a 469 bp product for the short allele and a 512 bp product for the long allele. Each PCR reaction consisted of $1 \times$ MyTaq Mix (Bioline, UK), $200 \mathrm{nM}$ each primer, and $50 \mathrm{ng}$ genomic DNA, to a final volume of $25 \mu \mathrm{L}$. The reaction was performed under the following conditions: 15 minute denaturation at $95^{\circ} \mathrm{C}$, followed by 35 cycles of $95^{\circ} \mathrm{C}$ (30 seconds), $65.5^{\circ} \mathrm{C}$ (90 seconds), and $72^{\circ} \mathrm{C}$ (60 seconds), followed by a final elongation step at $72^{\circ} \mathrm{C}$ for 10 minutes. Ten microliters of each PCR product was loaded on a $3 \%$ agarose gel containing GelRed (Biotium, USA), run for 1 hour at $100 \mathrm{~V}$ in TBE, and visualized under UV light. All genotyping reactions were completed in duplicate, with negative controls included in each assay.

\section{Procedure}

From a list of 20,000 random names and addresses (balanced for equal numbers of males and females) supplied by the Australian Electoral Commission in 2013, sufficient participants were recruited for this study to exceed the target of at least 60 participants in each of the two 5HTTLPR variant subsamples of s-present (defined as ss or sl) versus s-absent (defined as 11) to meet a priori power analysis requirements for correlation and multivariate analysis of variance 
(MANOVA) models. Participants received an online portal or a copy of the questionnaire booklet containing the background questionnaire and the SDS, Recent Life Stressors and Childhood Stressors scales, a small container half-filled with mouthwash for collection of buccal cells, as well as written instructions to tip the mouthwash into their mouth, vigorously swill the mouthwash around their mouth for 2 minutes, spit the mouthwash into the container without touching it with their fingers, and seal the container and place it in their home freezer $\left(-20^{\circ} \mathrm{C}\right)$, and post it back to the researchers or bring it to them. Participants' data were de-identified and analyzed through SPSS. The project was approved by the University of New England Human Research Ethics Committee. All participants gave written informed consent to the study.

\section{Statistical analyses}

Within studies of multiple dependent variables that are tested for their association with independent variables, the likelihood of a Type I error can increase because of multiple testing of effects. In this study, the investigation of the 20 SDS items represents such a situation where the likelihood of a Type I error might increase if $p<0.05$ were used to test for effects. It is commonplace to adjust this value according to some metric that seeks to counter the inflated family-wise error rate, often through a Bonferroni correction, where the 0.05 value is divided by the number of tests conducted. In this study, that figure would be $0.05 / 20=0.0025$. However, there is also a need to balance the likelihood of Type II error rates against Type I error rates, and it has also been argued that adjustment of the kind described above might unwisely inflate the likelihood of a Type II error, especially in exploratory studies (such as this one) where no a prior hypothesis is being tested. ${ }^{41}$ It has also been suggested that a general caution should be used when applying corrections to $p$-values when engaged in multiple variable testing, ${ }^{42}$ and so it was decided to not apply a stringent correction procedure (eg, Bonferroni adjustments) to the results of the SDS item analyses in this study but rather to provide a compromise adjustment of $0.05 / 10=0.005$.

\section{Results Data}

A total sample of 249 participants was recruited for this study (mean age $=53.6$ years, $\mathrm{SD}=17.2$ years, range $=18-82$ years), including 90 males (36.1\%) and 159 females (63.9\%). All raw data met the requirements for normality based upon the Kolgomorov-Smirnov statistic, and no transformations were required. MANOVA on age, SDS total score, Recent
Life Stressors, Childhood Stressors, and the distribution of the 5HTTLPR variants according to sex of respondents revealed no significant main effect $F(4,293)=2.380, p=0.052$, partial eta squared $=0.031$, and there were no significant correlations between age and any of these variables. The mean scores, standard deviations, and ranges for the scales are shown in Table 1, as well as the relative distributions of the two 5HTTLPR variants. There was a significant correlation between Recent Life Stress and SDS total score for the whole sample $(r=-0.271, p<0.001)$ but not between Childhood Stressors and SDS total score $(r=0.054, p=0.283)$.

Research questions 1 and 2: Is there a significant difference in the severity of total depression across participants carrying the s-present versus s-absent variants of the 5HTTLPR? And, is there a significant difference in the severity of individual depression items across participants carrying the s-present versus s-absent variants of the 5HTTLPR?

Using Pillai's trace because of the unequal prevalence of the two 5HTTLPR variants, ${ }^{43}$ there were no significant MANOVA main effects $F(20,228)=1.170, p=0.282$, partial eta squared $=0.093$ or univariate effects for the 20 SDS items across the two 5HTTLPR variants. These initial findings indicated that the presence of the 5HTTLPR s-variants was not significantly associated with elevated total or individual SDS item differences.

Research questions 3 and 4: Is there a difference in the association between total depression and total Recent Life Stressors or Childhood Stressors across participants carrying the s-present versus s-absent variants of the 5HTTLPR? And, is there a difference in the association between individual depression items and total Recent Life Stressors or Childhood Stressors across participants carrying the s-present versus s-absent variants of the 5HTTLPR?

Pearson's correlation coefficients were calculated separately for the associations between the total SDS score and Recent Life Stressors and Child Stressors for each set of the 5HTTLPR variants (ie, s-present versus s-absent). There were significant ( $p=0.05 / 2=0.025$ because of testing for both Recent Life Stressors and Childhood Stressors) associations between total SDS score and Recent Life Stressors $(r=0.237$, $p=0.001)$ and Childhood Stressors $(r=0.260, p<0.001)$ for the s-present participants or for the s-absent participants (Recent Life Stressors, $r=0.415, p=0.001$; Childhood Stressors, $r=0.516, p<0.001)$. There was no significant difference in the size of the correlation coefficients for the association between Recent Life Stressors and SDS total score across the two 5HTTLPR variants $(z=1.36, p=0.173)$, but the correlation 
Table 2 Significant ( $p \leq 0.005$ ) Pearson's correlations between SDS items and Recent Life Stress or Childhood Stress for the s-present subgroup $(n=184)$

\begin{tabular}{|c|c|c|c|c|c|}
\hline SDS items & $\begin{array}{l}\text { I feel downhearted } \\
\text { and blue }\end{array}$ & $\begin{array}{l}\text { Morning is when } \\
\text { I feel best }\end{array}$ & $\begin{array}{l}\text { I have crying spells } \\
\text { or feel like it }\end{array}$ & $\begin{array}{l}\text { My heart beats } \\
\text { faster than usual }\end{array}$ & $\begin{array}{l}\text { I get tired for no } \\
\text { reason }\end{array}$ \\
\hline Recent Life Stress & 0.224 & & 0.216 & 0.210 & 0.201 \\
\hline Childhood Stress & 0.229 & 0.204 & & & 0.253 \\
\hline
\end{tabular}

Abbreviation: SDS, Self-rating Depression Scale.

between Childhood Stressors and SDS total score for the $\mathrm{s}$-absent subgroup was significantly greater than that for the s-present subgroup ( $z=0.207, p=0.038$, two-tailed).

To test for these effects at the individual SDS item level, further Pearson's correlation coefficients were calculated between total Recent Life Stressor scores and the 20 SDS items, and then between total Childhood Stressor scores and the 20 SDS items for the s-present and s-absent subgroups. Tables 2 and 3 present the significant results from each of those correlational analyses, with the traditional $(p<0.05)$ level of significance adjusted to $p \leq 0.005$ to offer a balance between the likelihood of Type I and Type II errors due to multiple testing in this exploratory study.

To identify the key SDS items in these four sets of associations, hierarchical regression equations were calculated for each of Recent Life Stressors and Childhood Stressors for the s-present and s-absent subgroups. There were significant ANOVA effects for each of the four sets of regressions, and Table 4 shows those SDS items that made a significant contribution to the variance for Recent Life Stressors and Childhood Stressors for each 5HTTLPR variant subgroup. These findings are summarized graphically in Figure 1 to show more clearly the disparate associations that each source of stress had with the symptoms of depression measured by the SDS for the s-present and s-absent subgroups.

\section{Discussion}

There were no significant differences between the total depression scores of those participants who carried the s-variant of the 5HTTLPR versus those who did not, verifying the findings of Lopez-Lean et $\mathrm{al}^{11}$ and many individual studies reviewed in subsequent meta-analyses. In an extension to those previous findings, there were no significant differences in the individual SDS item scores across the two 5HTTLPR variant subgroups, further emphasizing the lack of a major effect of this genetic factor upon depression scores per se. However, the investigation of the nature of the $g \times e$ interaction in the form of the correlation matrices between SDS total scores, Recent Life Stressors, and Childhood Stressors produced some new findings.

First, the presence of significant associations between total SDS score and Recent Life Stressors, as well as between SDS total score and Childhood stressors for both of the 5HTTLPR variant subgroups, suggested that both variants were vulnerable to the effects of Recent Life Stressors and Childhood Stressors upon total depression scores. However, the significantly stronger correlation between Childhood Stressors and SDS total score for the s-absent subgroup than for the s-present subgroup suggested that there might be some variation in the ways that the $g \times$ interaction was manifested across the two 5HTTLPR subgroups. That variation was clarified by the correlation matrices shown in Table 4 and depicted graphically in Figure 1 when the individual items of the SDS were considered rather than the total SDS score.

It is apparent from Figure 1 that the $\mathrm{g} \times \mathrm{e}$ interaction for the s-present subgroup and Recent Life Stressors produced different symptoms of depression than that same interaction produced for the s-absent subgroup. These relationships might be explained in this way. The depressive effect of Recent Life Stressors upon carriers of the s variant was most powerfully felt in feelings of being downhearted and blue (ie, the "depressed mood" aspect of major depressive disorder symptomatology).

Table 3 Significant $(p \leq 0.005)$ Pearson's correlations between SDS items and Recent Life Stress or Childhood Stress for the s-absent subgroup $(n=65)$

\begin{tabular}{llllllll}
\hline SDS items & $\begin{array}{l}\text { I have crying } \\
\text { spells or feel } \\
\text { like it }\end{array}$ & $\begin{array}{l}\text { I have trouble } \\
\text { sleeping at } \\
\text { night }\end{array}$ & $\begin{array}{l}\text { My heart } \\
\text { beats faster } \\
\text { than usual }\end{array}$ & $\begin{array}{l}\text { I am restless } \\
\text { and cannot } \\
\text { keep still }\end{array}$ & $\begin{array}{l}\text { I am more } \\
\text { irritable than } \\
\text { usual }\end{array}$ & $\begin{array}{l}\text { I find it easy to } \\
\text { make decisions }\end{array}$ & $\begin{array}{l}\text { I feel that others } \\
\text { would be better } \\
\text { off if I were dead }\end{array}$ \\
\hline Recent Life Stress & 0.398 & 0.346 & 0.353 & 0.428 & & 0.367 & 0.5 I6 \\
Childhood Stress & $0.4 I 7$ & 0.344 & 0.453 & 0.423 & 0.424 & & \\
\hline
\end{tabular}

Abbreviation: SDS, Self-rating Depression Scale. 
Table 4 Significant SDS item predictors of Recent Life Stressors and Childhood Stressors for the s-present and s-absent subgroups

\begin{tabular}{|c|c|c|c|c|c|}
\hline $\begin{array}{l}\text { 5-HTTLPR } \\
\text { subgroup }\end{array}$ & Stressor source & $\begin{array}{l}\text { SDS items making a significant unique } \\
\text { contribution to variance }\end{array}$ & $R^{2}$ change & $\boldsymbol{F}$ & $p$-value \\
\hline \multirow[t]{4}{*}{ s-present } & Recent Life & I feel downhearted and blue & 0.050 & 9.634 & 0.002 \\
\hline & Stressors & & & & \\
\hline & Childhood & I get tired for no reason & 0.064 & 12.475 & 0.001 \\
\hline & Stressors & Morning is when I feel best & 0.022 & 4.437 & 0.037 \\
\hline \multirow[t]{4}{*}{ s-absent } & Recent Life & I am restless and cannot keep still & 0.116 & 8.847 & 0.004 \\
\hline & Stressors & I feel that others would be better off if I were dead & 0.069 & 4.671 & 0.034 \\
\hline & Childhood & My heart beats faster than usual & 0.205 & 16.254 & $<0.001$ \\
\hline & Stressors & I am more irritable than usual & 0.084 & 7.319 & 0.009 \\
\hline
\end{tabular}

Abbreviation: SDS, Self-rating Depression Scale.

By contrast, those participants who did not carry the $s$ variant were more likely to experience somatic (restlessness) and pessimistic (others would be better off if I were dead) symptoms of depression as a response to Recent Life Stressors. Of note, only one of these three MDD symptoms is a required symptom for that disorder (ie, depressed mood). In one way, this might be interpreted as suggesting that the s-carriers experienced a more severe type of depression when measured in terms of the actual symptoms experienced.

There were also differences in the way that the two 5HTTLPR subgroups experienced depression as associated with Childhood Stressors. Carriers of the s variant felt fatigued and also exhibited one of the key characteristics of melancholic depression ${ }^{3}$ in their reports that they felt best in the morning. Melancholia is one of the most difficult-totreat forms of depression, ${ }^{3}$ and this symptom is considered to reflect disruption to the circadian rhythm. ${ }^{44}$ It has been suggested that this kind of disruption to one of the most central aspects of human physiology represents a fundamental challenge to normal functioning in persons who exhibit it. ${ }^{45}$ As such, the presence of disruption to circadian rhythm may represent a serious clinical management issue for people

A

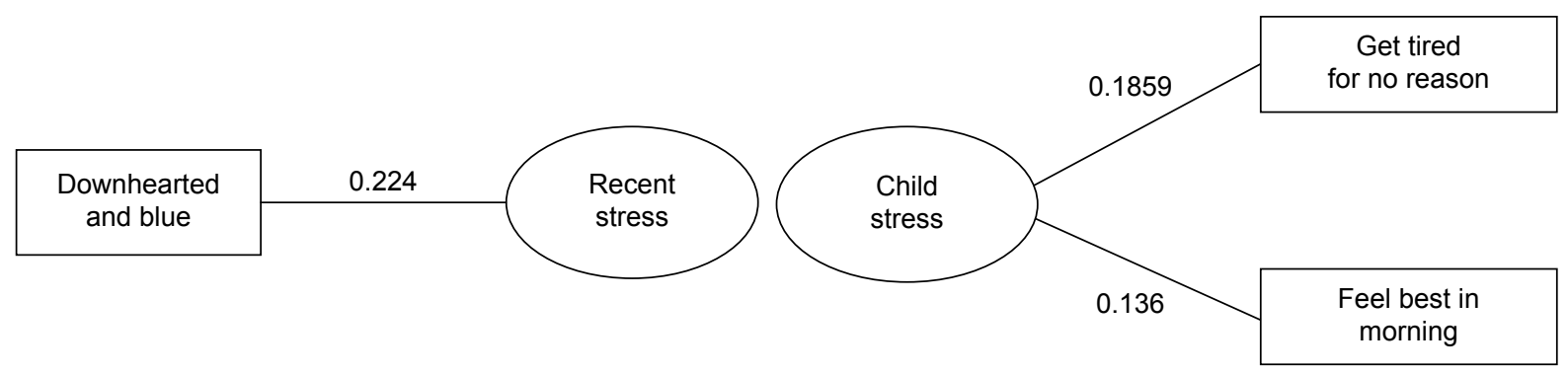

B

s-absent

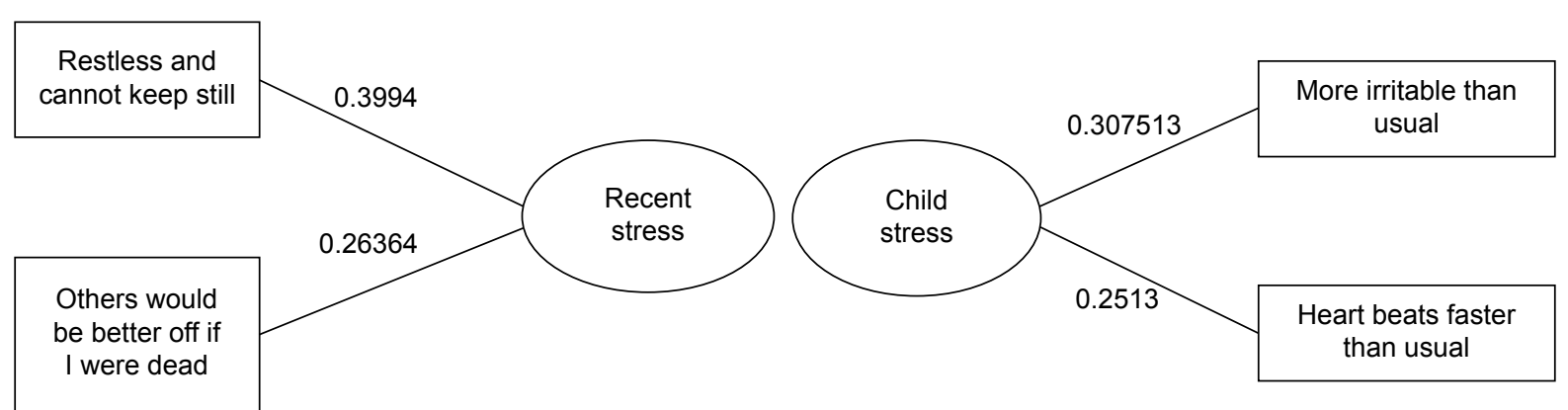

Figure I Major (beta weights) SDS items contributing to Recent Life Stressor and Childhood Stressor scores for participants carrying 5 HTTLPR s-variant versus participants not carrying 5HTTLPR s-variant. $\mathbf{A}=\mathrm{s}$ is present; $\mathbf{B}=\mathrm{s}$ is absent.

Abbreviation: SDS, Self-rating Depression Scale. 
who exhibit it. By comparison, those participants who did not carry the $s$ variant showed an association between Childhood Stressors and irritability, as well as sympathetic arousal (increased heart rate). Although these are considerable symptoms of depression, they are not as serious as the disruption to circadian rhythm and may provide another indication of the more severe nature of depression as it is experienced by carriers of the $s$ variant who have also undergone childhood stressors.

Therefore, these findings may represent a different method of understanding the $\mathrm{g} \times \mathrm{e}$ interaction across the 5HTTLPR variants, with some suggestions that those participants who carried the $s$ variant experienced more severe types of depression when considered from the standpoint of the types of symptoms that were associated with stressor events rather than the depression inventory scores alone. This finding is new and must be considered as tentative at this stage, requiring confirmation across larger samples, but it does represent a more detailed depiction of the 5HTTLPR-stressdepression association than has been reported previously, potentially accounting for at least some of the inconsistencies in the previous literature.

\section{Clinical implications}

Clinical implications arising from these findings are also tentative at this stage but do suggest considering the presence of the $s$ variant of the 5HTTLPR as a potential contributor to the risk of more severe depression symptomatology. Clinicians who may not detect any significant difference in the total depression inventory scores of s-present versus s-absent patients may find the exploration of specific MDD symptom profiles of benefit in identifying the presence of more-difficult-to-treat profiles in their patients. The application of a "one-size-fits-all" treatment protocol has been shown to be less than ideal in the STAR*D study, ${ }^{4}$ and the results of the present study provide support for the development of treatment models that are designed to individualize therapy. Taking the current findings of presence of a major symptom of melancholic depression among those $s$ variant carriers who had also experienced Childhood Stressors, some data suggest that depressed persons who exhibit melancholic features are more likely to attempt violent suicide, ${ }^{46}$ and there is a longstanding clinical belief that patients with melancholic depression respond better to pharmacologic treatments than to psychotherapy, ${ }^{47}$ presumably because of the powerful biologic underpinnings to their depression. These factors are worth inclusion in treatment planning processes for patients who exhibit symptoms of melancholia, and it may be that carriage of the $\mathrm{s}$ variant of the 5HTTLPR increases that risk.

\section{Study limitations}

Limitations of this study include the sample size, cultural and geographic specificity of the sample, the "snapshot" nature of the study, and the use of self-report inventories to assess depression and stressor experiences. Collection of data from other samples, using a prospective design to detect the effects of specific stressors upon depressive symptomatology profiles, and using clinician interviews and observations to triangulate self-report data would all add to the generalizability of the current findings. Notwithstanding those suggestions, the SDS is very well validated across multiple populations and has high cross validity with clinician diagnoses (mentioned under the "Materials and methods" section), and so may be accepted as providing a reasonably accurate dataset for the depressive symptomatology of the sample.

\section{Conclusion}

Effective research and treatment of depression are predicated upon accurate identification of depressed persons, the severity of their depression, and the ways in which the depression they experience influences their physical and mental health. When combined with the finding that only about one-third of persons diagnosed with depression receive treatment, ${ }^{48}$ the serious effects that depression has upon daily functioning, cost of care, and personal distress that were mentioned above, as well as the fact that many people with depression find themselves in a chronic state rather than experiencing a passing condition (as evidenced by the finding that depression has a longitudinal stability over a 15-year period ${ }^{49}$ ), depression clearly ranks very highly in terms of worthwhile targets for investigation and treatment. Identification of persons who may be more at risk for particular depression symptoms represents a valuable addition to standard clinical care and a valuable step toward individualizing therapy.

\section{Acknowledgments}

The authors express their gratitude to all the participants who took part in this study.

\section{Author contributions}

All Authors contributed toward data analysis, drafting and revising the paper and agree to be accountable for all aspects of the work. 


\section{Disclosure}

The authors report no conflicts of interest in this work.

\section{References}

1. Vos T, Flaxman AD, Naghavi M, et al. Years lived with disability (YLDs) for 1160 sequelae of 289 diseases and injuries 1990-2010: a systematic analysis for the Global Burden of Disease Study 2010. Lancet. 2012;380(9859):2163-2196.

2. Moussavi S, Chatterji S, Verdes E, Tandon A, Patel V, Ustun B. Depression, chronic diseases, and decrements in health; results from the World Health Surveys. Lancet. 2007;370(9590):851-858.

3. American Psychiatric Association. Diagnostic and Statistical Manual of Mental Disorders-5. Washington, DC: American Psychiatric Association; 2013

4. Warden D, Rush AJ, Trivedi MH, Fava M, Wisniewski SR. The STAR*D Project results: a comprehensive review of findings. Curr Psychiatry Rep. 2007;9(6):449-459.

5. Rush AJ, Trivedi MH, Wisniewski SR, et al. Acute and longer-term outcomes in depressed outpatients requiring one or several treatment steps: a STAR* report. Am J Psychiatry. 2006;163(11):1905-1917.

6. Wallace ML, Frank E, Kraemer HC. A novel approach for developing and interpreting treatment moderator profiles in randomized clinical trials. JAMA Psychiatry. 2013;70(11):1241-1247.

7. Otte C, Gold SM, Penninx BW, et al. Major depressive disorder. Nat Rev Dis Primers. 2016;2:16065.

8. Heils A, Teufel A, Petri S, et al. Allelic variation of human transporter gene expression. J Neurochem. 1996;66(6):2621-2624.

9. Lesch KP, Bengel D, Heils A, et al. Association of anxiety-related traits with a polymorphism in the serotonin transporter gene regulatory region. Science. 1996;274(5292):1527-1531.

10. Caspi A, Sugden K, Moffitt TE, et al. Influence of life stress on depression: moderation by a polymorphism in the 5-HTT gene. Science 2003;301(5631):386-389.

11. Lopez-Leon S, Janssens AC, Gonzalez-Zuloeta Ladd AM, et al. Metaanalysis of genetic studies on major depressive disorder. Mol Psychiatry. 2008;13(8): 772-785.

12. Risch N, Herrell R, Lehner T, et al. Interaction between the serotonin transporter gene (5-HTTLPR), stressful life events, and risk of depression: a meta-analysis. JAMA. 2009;301(23):2462-2471.

13. Munafo MR, Durrant C, Lewis G, Flint J. Gene X environment interaction at the serotonin transporter locus. Biol Psychiatry. 2009;65(3): 211-219.

14. Uher R, McGuffin $P$. The moderation by the seritonin transporter gene of environmental adversity in the etiology of depression: 2009 update. Mol Psychiatry. 2010;15(1):18-22.

15. Karg K, Burmeister M, Shedden K, Sen S. The serotonin transporter promoter variant (5-HTTLPR), stress, and depression meta-analysis revisited: evidence of genetic moderation. Arch Gen Psychiatry. 2011; 68(5):444-454.

16. Duncan LE, Keller MC. A critical review of he first 10 years of candidate gene-by-environment interaction research in psychiatry. $\mathrm{Am} \mathrm{J}$ Psychiatry. 2011;168(10):1041-1049.

17. Sharpley CF, Palanisamy SK, Glyde NS, Dillingham PW, Agnew LL. An update on the interaction between the serotonin transporter promoter variant (5-HTTLPR), stress and depression, plus an exploration of nonconfirming findings. Behav Brain Res. 2014;273:89-105.

18. Bleys D, Luyten P, Soenens B, Claes S. Gene-environment interactions between stress and 5-HTTLPR in depression: a meta-analytic update. J Affect Disord. 2018;226:339-345.

19. Culverhouse RC, Saccone NL, Horton AC, et al. Collaborative metaanalysis finds no evidence of a strong interaction between stress and 5-HTTLPR genotype contributing to the development of depression. Mol Psychiatry. 2017;23(1):133-142.

20. Ostergaard SD, Jensen SO, Bech P. The heterogeneity of the depressive syndrome: when numbers get serious. Acta Psychiatr Scand. 2011;124(6) 495-496.
21. Fried E, Nesse RM. Depression is not a consistent syndrome: an investigation of unique symptom patterns in the STAR*D study. $J$ Affect Disord. 2015;172:96-102.

22. Sutin AR, Terracciano A, Milaneschi Y, An Y, FerrucciL, Zonderman AB. The trajectory of depressive symptoms across the adult life span. JAMA Psychiatry. 2013;70(8):803-811.

23. Insel T. Transforming diagnosis; 2013 Post. Available from: http:// www.nimh.nih.gov/about/director/2013/transforming-diagnosis-shtml. Accessed May 27, 2013.

24. Cohen J. The cost of dichotomization. Appl Psychol Meas. 1983;7: 249-253.

25. Caldwell TM, Jorm AF, Dear KB. Suicide and mental health in rural, remote and metropolitan areas in Australia. Med J Aust. 2004; 181(7 Suppl):S10-S14.

26. Kilkkinen A, Kao-Philpot A, O'Neil A, et al. Prevalence of psychological distress, anxiety and depression in rural communities in Australia. Aust J Rural Health. 2007;15(2):114-119.

27. Centre for Rural and Remote Mental Health. Suicide \& Suicide Prevention in Rural Areas of Australia: Briefing Paper. Rural Suicide Prevention Forum; April 11th, 2017; Orange, New South Wales, Australia.

28. Gokturk C, Schultze S, Nilsson KW, von Knorring L, Oreland L, Hallman J. Serotonin transporter (5-HTTLPR) and monoamine oxidase (MAOA) promoter polymorphisms in women with severe alcoholism. Arch Womens Ment Health. 2008;11(5-6):347-355.

29. Brummett BH, Boyle SH, Siegler IC, et al. Effects of environmental stress and gender on associations among symptoms of depression and the serotonin transporter gene linked polymorphic region (5-HTTLPR). Behav Genet. 2008;38(1):34-43.

30. Zung WW. A self-rating depression scale. Arch Gen Psychiatry. 1965;12: 63-70.

31. American Psychiatric Association. Diagnostic and Statistical Manual of Mental Disorders (4th ed, Text Revision). Washington, DC: American Psychiatric Association; 2000

32. Zung WW. From art to science. The diagnosis and treatment of depression. Arch Gen Psychiatry. 1973;29(3):328-337.

33. deJonge JFM, Baneke JJ. The Zung Self-rating Depression Scale: a replication study on reliability, validity and prediction. Psychol Rep. 1989;64:833-834.

34. Gabrys JB, Peters K. Reliability, discriminant and predictive validity of the Zung Self-Rating Depression Scale. Psychol Rep. 1985;57(3 Pt 2): 1091-1096.

35. Schaefer A, Brown J, Watson CG, et al. Comparison of the validities of the Beck, Zung and MMPI depression scales. J Consult Clin Psychol. 1985; 53(3):415-418.

36. Sharpley CF, Bitsika V, Christie DR. Understanding the causes of depression among prostate cancer patients: development of the effects of prostate cancer on lifestyle questionnaire. Psycho Oncol. 2009;18(2): $162-168$.

37. Schilling EA, Aseltine RH, Gore S. The impact of cumulative childhood adversity on young adult mental health: measures, models and interpretations. Soc Sci Med. 2008;66(5):1140-1151.

38. Kessler RC, Zhao S, Blazer DG, Swartz M. Prevalence, correlates, and course of minor depression and major depression in the national comorbidity survey. J Affect Disord. 1997;45(1-2):19-30.

39. Hampson SE, Goldberg LR, Vogt TM, Dubanoski JP. Mechanisms by which childhood personality traits influence adult health status: educational attainment and healthy behaviors. Health Psychol. 2007;26(1):121-125.

40. Wendland JR, Martin BJ, Kruse MR, Lesch KP, Murphy DL. Simultaneous genotyping of four functional loci of human SLC6A4, with a reappraisal of 5-HTTLPR and rs25531. Mol Psychiatry. 2006;11(3): 224-226.

41. Rothman KJ. No adjustments are needed for multiple comparisons. Epidemiology. 1990;1(1):43-46.

42. Streiner DL, Norman GR. Correction for multiple testing: is there a resolution? Chest. 2011;140(1):16-18.

43. Tabachnik B, Fidell L. Using Multivariate Statistics. 6th ed. Boston, MA: Pearson Education; 2013. 
44. Germain A, Kupfer DJ. Circadian rhythm disturbances in depression. Hum Psychopharmacol. 2008;23(7):571-585.

45. Levitan RD. The chronobiology and neurobiology of winter seasonal affective disorder. Dialogues Clin Neurosci. 2007;9(3):315-324.

46. Van Gastel A, Schotte C, Maes M. The prediction of suicide intent in depressed patients. Acta Psychiatr Scand. 1997;96(4):254-259.

47. Feinberg M. Subtypes of depression and response to treatment. J Consult Clin Psychol. 1992;60(5):670-674.
48. Kocsis JH, Gelenberg AJ, Rothbaum BO, et al. Chronic forms of major depression are still undertreated in the 21th centry: systematic assessment of 801 patients presenting for treatment. J Affect Disord. 2008;110(1-2): $55-61$.

49. Merikangas KR, Zhang H, Avenoli S, Acharyya A, Neuenschwander M, Angst J; Zurich Cohort Study. Longitudinal trajectories of depression and anxciety in a prospective community study. Arch Gen Psychiatry. 2003;60(10):993-1000.

\section{Publish your work in this journal}

Neuropsychiatric Disease and Treatment is an international, peerreviewed journal of clinical therapeutics and pharmacology focusing on concise rapid reporting of clinical or pre-clinical studies on a range of neuropsychiatric and neurological disorders. This journal is indexed on PubMed Central, the 'PsycINFO' database and CAS, and is the official journal of The International Neuropsychiatric Association (INA). The manuscript management system is completely online and includes a very quick and fair peer-review system, which is all easy to use. Visit http://www.dovepress.com/testimonials.php to read real quotes from published authors.

Submit your manuscript here: http://www.dovepress.com/neuropsychiatric-disease-and-treatment-journal 